\title{
Exploration of miR-1202 and miR-196a in human endometrial cancer based on high throughout gene screening analysis
}

\author{
HONG CHEN, YUJUAN FAN, WENSHENG XU, JUNYING CHEN, YUGANG MENG, \\ DI FANG and JINGRAN WANG \\ Department of Gynaecology, The First Affiliated Hospital of Guangxi Medical University, \\ Nanning, Guangxi 530021, P.R. China
}

Received August 25, 2016; Accepted November 18, 2016

DOI: $10.3892 /$ or.2017.5596

\begin{abstract}
Altered microRNA (miRNA) expression has been reported to participate in the pathogenesis of several human diseases, and particularly cancer. The present study examined the involvement of various miRNAs in the pathophysiology of endometrial cancer (EC) and atypical endometrial hyperplasia (AEH). We performed a high-throughput analysis of the miRNAs (miRNA microarray) found in samples of endometrial tissue obtained from 45 patients; among whom, 15 patients were diagnosed with EC, 15 patients were diagnosed with $\mathrm{AEH}$, and the remainder were healthy donors. Next, we selected several miRNAs which exhibited at least a 2-fold difference in expression with a $\mathrm{P}<0.05$ to validate these changes in 3 independent in vitro experiments that used real-time PCR analysis. Finally, miR-1202 and miR-196a were selected as target molecules whose effects on cell apoptosis, cell cycle changes, cell migratory and invasive abilities were investigated using flow cytometric and Transwell assays, respectively, after pre-treatment in vitro. After analyzing 125 miRNAs in a microarray assay, 6 miRNAs (3-high and 3-low expression) were further evaluated via paired comparison in all 3 groups. The validation test revealed a positive correlation between the microarray results and a high level of miR-1202 and a low level of miR-196a in the EC group, when compared with the AEH group. All of the data were normalized with data obtained from normal control donors. We found that either miR-1202 silencing or miR-196a overexpression affected AN3CA and HEC-1-A cells by increasing their apoptosis level and inducing G1 phase arrest while decreasing their migratory and invasive abilities. Inhibitors of miR-1202 and mimics of miR-196a may exert a protective effect, suggesting that miR-1202 and miR-196a may serve as biomarkers for evaluating the effectiveness of EC treatment.
\end{abstract}

Correspondence to: Dr Hong Chen, Department of Gynaecology, The First Affiliated Hospital of Guangxi Medical University, 6 Shuangyong Road, Nanning, Guangxi 530021, P.R. China

E-mail: doctorchen2016@hotmail.com; chenhong20090909@qq.com

Key words: endometrial cancer, atypical endometrial hyperplasia, miRNA, microarray, validation test, cell function test

\section{Introduction}

Endometrial cancer (EC) is the fourth most common malignancy among postmenopausal females in the developed world, and its incidence is surpassed only by lung, colorectal and breast cancer (1-3). Although, EC accounts for 74,000 deaths each year worldwide, the majority of affected women have a good prognosis, as abnormal vaginal bleeding begins at an early stage of the disease, and thus, the 5-year survival rate is 80-82\% (4). Atypical endometrial hyperplasia (AEH) is a type of pre-cancerous lesion which is a significant risk factor for the development or co-existence of EC. However, traditional surgical resection, the standard method for treating EC and $\mathrm{AEH}$ is a total hysterectomy, such as a bilateral oophorectomy, which results in a total loss of fertility. As a result, this method of treatment is unacceptable to many women diagnosed with EC or AEH $(5,6)$. Molecular-targeted therapies for EC, such as microRNA (miRNA)-based therapies, have received increased attention, even though the specific molecular events which lead to EC development remain unclear. However, various studies have investigated the molecular changes which lead to AEH (7-9). Research regarding the functions of miRNAs may increase our understanding of disease pathogenesis and particularly the pathogenesis of cancer.

miRNAs consist of a family of single-stranded, 22 nucleotide, non-coding, evolutionarily conserved RNAs, which regulate gene degradation or translational suppression at the post-transcriptional level by binding to the 3'-untranslated region (3'-UTR) of mRNAs $(10,11)$. The relevance of miRNAs in cancers such as EC is associated with their ability to regulate gene expression and various cellular processes, such as cell proliferation, differentiation, apoptosis, epigenetic dysfunction and carcinogenesis. These regulatory abilities suggest that miRNA expression may be important when developing a prognosis and treatment strategy for EC patients (12-14). Numerous studies have shown the importance of miRNAs, and miRNA profiling analyses have revealed significant variations in miRNA expression across different cancer subtypes and stages of carcinogenesis. Such findings suggest that miRNAs play important roles in the initiation and progression of human malignancies (15-17).

The exact biological functions of miRNAs in EC remain unclear. In the present study, we profiled miRNA expression in 
cases of human EC, and focused on the relationships between certain miRNAs that exhibited aberrant expression and the presence of EC, in order to explore the effect of those miRNAs on cellular functions and regulatory mechanisms. AN3CA and HEC-1-A cell lines were used in the present study, since HEC-1-A and AN3CA are both human EC cell lines that have been commonly used in EC research in vitro.

\section{Materials and methods}

Patient characteristics and sample collection. Between October 2015 and May 2016, 45 consecutive patients (15 with EC, 15 with AEH and 15 healthy donors) at The First Affiliated Hospital of Guangxi Medical University were recruited to participate in the present study. The EC patients were aged between 40 and 82 years, and had undergone a hysterectomy, bilateral salpingo-oophorectomy, pelvic and/or para-aortic lymphadenectomy or peritoneal washing for cytology.

Frozen fresh tissue sections and respective blood samples were obtained from 15 patients with EC, 15 patients with AEH, and 15 subjects with a normal endometrium. The samples were used in a microarray assay and also analyzed by quantitative real-time PCR. No patient had a history of adjuvant or neoadjuvant therapy prior to surgery. The study protocol was approved by the Ethics Committee of The First Affiliated Hospital of Guangxi Medical University, and each enrolled subject provided their signed informed consent for participation.

Profiling of miRNA expression. An Agilent Human miRNA Microarray kit (release 16.0; Agilent Technologies, Santa Clara, CA, USA) containing probes for 1,205 human miRNAs and 144 human viral miRNAs was used for miRNA expression profiling. The miRNA assays were performed according to the manufacturer's instructions. In brief, $100 \mathrm{ng}$ of total RNA from each sample was dephosphorylated and then ligated with $\mathrm{pCp}-\mathrm{Cy} 3$ dye. The labeled RNA was purified using a Micro Bio-SpinMicro Bio-Spin 66 column (Bio-Rad, Hercules, CA, USA), and then added to the miRNA array, which contained a hybridization buffer. After $20 \mathrm{~h}$ of incubation at $55^{\circ} \mathrm{C}$, the array slides were washed and scanned, and the images were analyzed using Feature Extraction 10.7.3.1 software (Agilent Technologies). Data quality was evaluated using an Agilent microRNA Spike-In kit, and all samples satisfied the Spike-In QC criteria (LabelingSpike-InSignal >2.5 and HybSpike-InSignal $>2.5$ ). The relevant miRNA microarray data is available in the NCBI Gene Expression Omnibus (GSE70574).

Real-time polymerase chain reaction. Total RNA was extracted using UNIQ-10 columns and a TRIzol Total RNA Isolation kit (Sangon, Shanghai, China). Cloned AMV reverse transcriptase (Invitrogen, Carlsbad, CA, USA) was used to reverse transcribe $1 \mu \mathrm{g}$ samples of total RNA in a reaction volume of $20 \mu$ l. Two microliters of cDNA were used for real-time PCR that was performed using a Takara Ex Taq RT-PCR version 2.1 kit (Takara, Shiga, Japan). Real-time quantification of mature miRNAs was performed using an Applied Biosystems 7500 Sequence Detection system (Applied Biosystems, Foster City, CA, USA). Each $20 \mu \mathrm{l}$ PCR reaction mixture contained $1 \mu \mathrm{l}$ of
RT product (1:5 dilution), $0.5 \mu 1$ of universal reverse primer, $0.5 \mu 1$ of sense primer, and $10 \mu \mathrm{l}$ of mix buffer (DBI Bestar ${ }^{\circledR}$ SybrGreen qPCR mastermix). The reaction mixtures were incubated in a 96 -well optical plate at $94^{\circ} \mathrm{C}$ for $2 \mathrm{~min}$, followed by 40 cycles of $94^{\circ} \mathrm{C}$ for $20 \mathrm{sec}, 58^{\circ} \mathrm{C}$ for $20 \mathrm{sec}$, and $72^{\circ} \mathrm{C}$ for $20 \mathrm{sec}$. All reactions were run in triplicate. The gene-specific miRNA primers are listed in Table I.

Cell line and culture conditions. Human endometrial cancer cell line AN3CA and HEC-1-A were purchased from the American Type Culture Collection (ATCC; Manassas, VA, USA), and maintained in Dulbecco's modified Eagle's medium (DMEM) containing 10\% fetal bovine serum (FBS), $100 \mathrm{U} / \mathrm{ml}$ penicillin, and $100 \mathrm{U} / \mathrm{ml}$ streptomycin in a humidified atmosphere of $5 \% \mathrm{CO}_{2}$ at $37^{\circ} \mathrm{C}$. The medium was changed every 2 or 3 days based on the recommended culture condition. All cells were harvested by centrifugation before being washed with phosphate-buffered saline (PBS) and subjected to total protein or RNA extraction.

Cells were cultured to $60-70 \%$ confluence, and then re-suspended in serum-free DMEM at a concentration of $10^{5}$ cells $/ \mathrm{ml}$. Six-well plates were inoculated with $2 \mathrm{ml}$ of cell suspension in each well, and 3 replicate wells were created for each experimental group. miR-1202 inhibitors and miR-196a mimics purchased from GenePharma (Shanghai, China) were diluted to $5 \mathrm{nM}$ concentrations with $0.25 \mathrm{ml}$ serum-free DMEM for use in transfection studies. Lipofectamine 2000 (Invitrogen) transfection reagent $(5 \mu \mathrm{l})$ was diluted with $0.25 \mathrm{ml}$ serum-free DMEM. Next, the diluted transfection reagent was added to the diluted mimics, mixed gently, and incubated for $20 \mathrm{~min}$ at room temperature. The cell suspension was refreshed with new medium, and then added to the mixture of Lipofectamine 2000 and the mimics aforementioned; after which, the total mixture was incubated at $37^{\circ} \mathrm{C}$ in a $5 \% \mathrm{CO}_{2}$ atmosphere for $6 \mathrm{~h}$. Subsequently, the medium in each well was replaced with normal serum-containing medium and incubated for $48 \mathrm{~h}$ prior to use in the following experiments.

Flow cytometric assay. After transfection for $72 \mathrm{~h}$, the apoptotic cells were quantified using an Annexin V/propidium iodide (PI) apoptosis kit (MultiSciences, Hangzhou, China) prior to performing cell apoptosis and cell cycle analyses. The AN3CA or HEC-1-A cells were collected, rinsed with PBS, and then resuspended in $200 \mu \mathrm{l}$ of binding buffer containing $5 \mu \mathrm{l}$ Annexin $\mathrm{V}(10 \mu \mathrm{g} / \mathrm{ml})$ for $10 \mathrm{~min}$ in the dark. After being incubated with $10 \mu \mathrm{l}$ of PI $(20 \mu \mathrm{g} / \mathrm{ml})$, the cells were immediately analyzed by flow cytometry (Beckman Coulter Epics XL; Beckman Coulter, Brea, CA, USA). Data acquisition and analysis were performed using CellQuest software (Becton-Dickinson, Franklin Lakes, NJ, USA).

Cell migration and invasion assays. Cell migration and invasion assays were performed in 24-well, Matrigel-coated invasion chambers. For this assay, AN3CA/HEC-1-A (non-transfected), AN3CA/HEC-1-A-NC and AN3CA/HEC-1-A-miR-1202 inhibitor/miR-196a mimic cells were plated at a density of $1.0 \times 10^{5}$ cells/well in wells containing $0.5 \mathrm{ml}$ of serum-free medium and polycarbonate filters $(8-\mu \mathrm{m}$ pore size; Costar Inc., Milpitas, CA, USA). The outer chambers were filled with $0.5 \mathrm{ml}$ of the medium supplemented with $10 \%$ FBS. 
Table I. Primer sequences used for miRNA expression analysis.

\begin{tabular}{lll}
\hline Gene & & \\
\hline U6 & F & CTCGCTTCGGCe $\left(5^{\prime}-3^{\prime}\right)$ \\
U6 & R & AACGCTTCACGAATTTGCGT \\
miR-1202 & RT & CTCAACTGGTGTCGTGGAGTCGGCAATTCAGTTGAGCTCCCC \\
miR-1202 & F & ACACTCCAGCTGGGGTGCCAGCTGCAGTGGG \\
miR-5787 & RT & CTCAACTGGTGTCGTGGAGTCGGCAATTCAGTTGAGACCTCC \\
miR-5787 & F & ACACTCCAGCTGGGGGGCTGGGGCGCGGGG \\
miR-6749-5p & RT & CTCAACTGGTGTCGTGGAGTCGGCAATTCAGTTGAGGCTCCC \\
miR-6749-5p & F & ACACTCCAGCTGGGTCGGGCCTGGGGTTGGG \\
miR-196a-5p & RT & CTCAACTGGTGTCGTGGAGTCGGCAATTCAGTTGAGCCCAAC \\
miR-196a-5p & F & ACACTCCAGCTGGGTAGGTAGTTTCATGTTG \\
miR-338-3p & RT & CTCAACTGGTGTCGTGGAGTCGGCAATTCAGTTGAGCAACAA \\
miR-338-3p & F & ACACTCCAGCTGGGTCCAGCATCAGTGATTTT \\
miR-449a & RT & CTCAACTGGTGTCGTGGAGTCGGCAATTCAGTTGAGACCAGC \\
miR-449a & F & ACACTCCAGCTGGGTGGCAGTGTATTGTTAGC \\
ALL & R & CTCAACTGGTGTCGTGGA \\
\hline
\end{tabular}

F, forward; R, reverse; RT, reverse transcription.
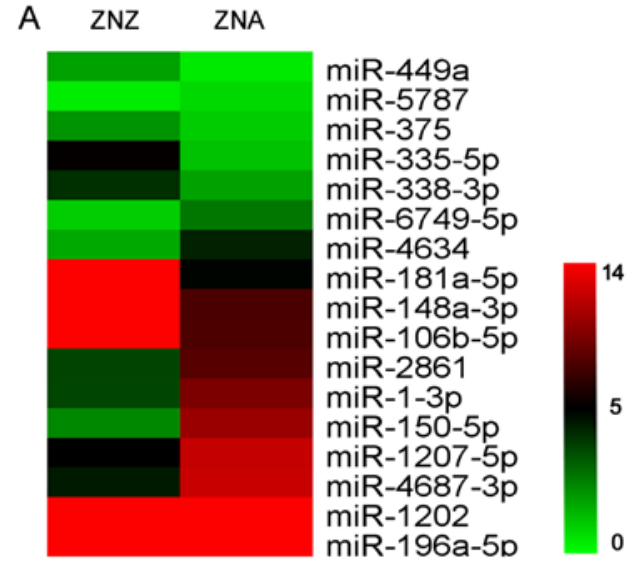

C ZZN ZNZ
B

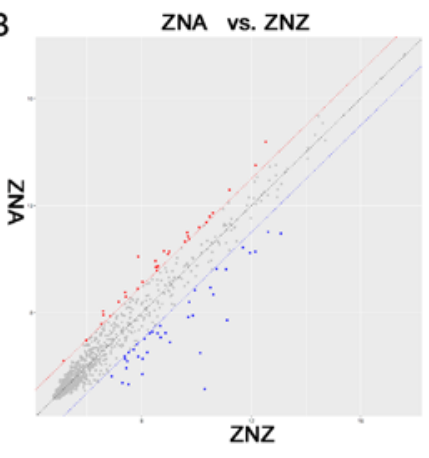

$\mathrm{D}$

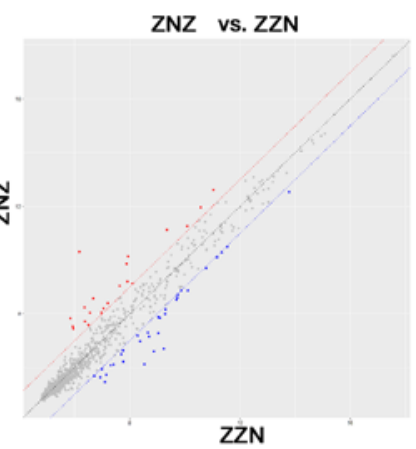

Figure 1. Cluster analysis of miRNA expression in tissue samples screened by miRNA microarray methodology. (A and C) Heatmaps displaying different hsamiRNAs on the the horizontal axis, with 2 columns for different cohorts. Red indicates upregulated hsa-miRNAs, green indicates downregulated hsa-miRNAs, and black indicates hsa-miRNAs that were not differentially expressed. Color shades represent the intensity of fluorescence and reflect the level of hsa-miRNA expression. The scheme indicates some obvious clustering properties of gene expression in EC patients. (B and D) Scatter plots showing clustering of hsa-miRNA expression in tissue samples, with a paired comparison in all 3 groups. The $\mathrm{x}$-axis is the fluorescence intensity value of cohort $\mathrm{N}$ [probe cohort in ZNZ (B) or ZZN (D)]; the y-axis is the fluorescence intensity value of cohort T [probe cohort in ZNA (B) or ZNZ (D)]. Each data point represents hybridization signals from a gene spot on the chip. ZNA, tissues from EC patients; ZNZ, tissues from EC patients with AEH; ZZN, tissues from healthy donors. All results were normalized with results from the healthy donor group. EC, endometrial cancer; $\mathrm{AEH}$, atypical endometrial hyperplasia. 
A

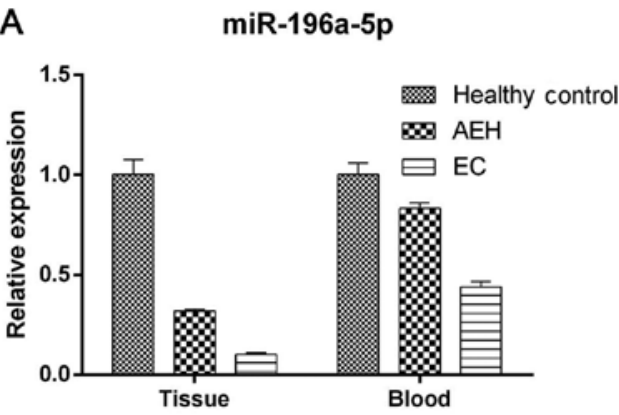

C

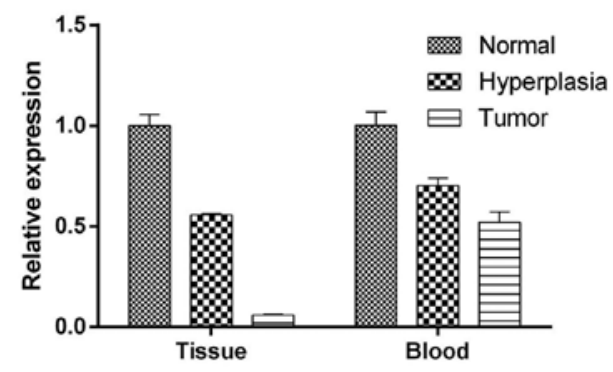

E

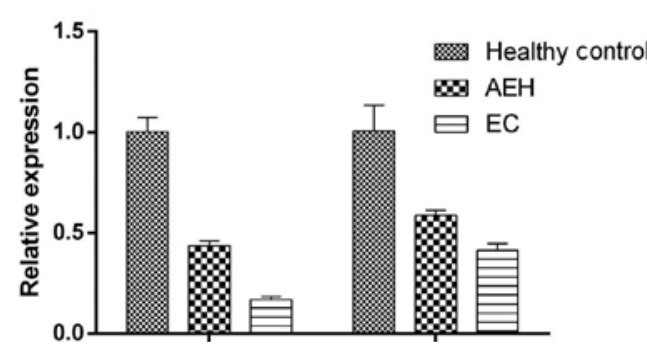

B

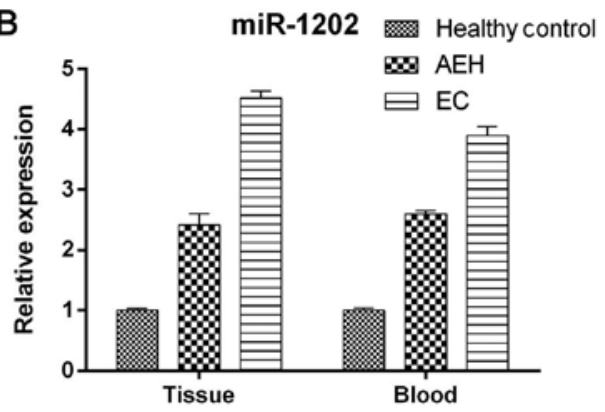

D

$\operatorname{miR}-5787$

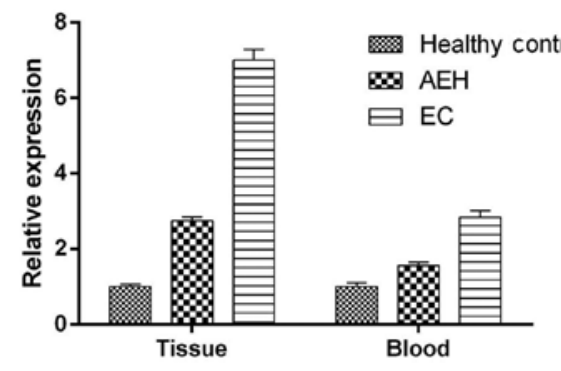

$\mathbf{F}$

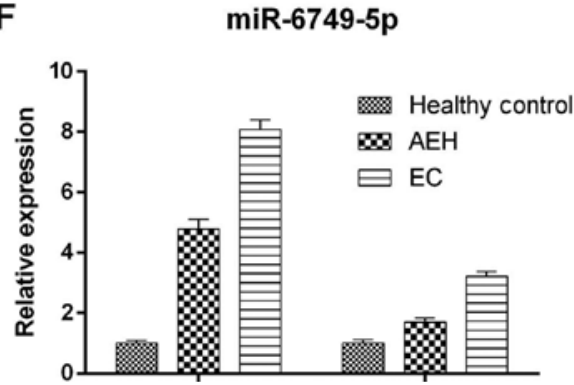

Figure 2. Validation of 6 hsa-miRNAs by real-time PCR analysis of tissue and blood samples, respectively. (A, C and E) Validation test results for 3 miRNAs with low expression in tissue and blood samples, respectively. (B, D and F) Validation test results for 3 miRNAs that were highly expressed in tissue and blood samples, respectively. Data are expressed as the mean \pm standard deviation. All data represent results from 3 independent experiments. EC, endometrial cancer; AEH, atypical endometrial hyperplasia.

After $24 \mathrm{~h}$, the cells were fixed in methanol and stained with crystal violet. Subsequently, the top surface of the membrane was gently scrubbed with a cotton bud, and the cells that had invaded through the membrane filters were counted. The invasion inhibition rate (\%) was calculated as [(A - B)/A) x 100]; where $\mathrm{A}$ and $\mathrm{B}$ are the percentages of invading cells for the miRNA inhibitor group and normal control (NC) group, respectively. Each experiment was performed in triplicate.

Statistical analysis. The miRNA array data were processed by quantile normalization, followed by a $\log 2$ transformation. The spots called 'absent' by the Agilent Feature Extraction software were discarded. The unpaired Mann-Whitney test was used to identify significant differences in expressed miRNA between LNM-positive and -negative CRC samples. The Benjamini-Hochberg false discovery rate (FDR) method was used for multiple comparison corrections. miRNAs with an FDR $<0.1$ and log fold-change $>3$ were considered as potentially important and included in further independent replication experiments performed for validation purposes. All data were analyzed using GeneSpring 12.6 software (Agilent Technologies).
All other data were analyzed using SPSS Statistics for Windows, version 17.0. (SPSS, Inc., Chicago, IL, USA) and the presence of a normal data distribution was assessed by the Kolmogorov-Smirnov test. miRNA expression results for the 3 groups of tissue are presented as the mean \pm standard deviation. Differences between groups were evaluated using one-way ANOVA for 3-group comparisons and t-tests for 2-group comparisons.

\section{Results}

Differentially expressed miRNAs are found in endometrial adenocarcinoma and AEH tissues when compared with normal endometrial tissue. We performed miRNA analyses to examine the global miRNA expression profiles in tissue samples obtained from 15 EC patients, 15 patients with $\mathrm{AEH}$, and 15 healthy donors. A paired comparison analysis identified 34 miRNAs that were expressed at significantly different levels in the 3 groups. Among these differentially expressed miRNAs, 14 were upregulated and 20 were downregulated in EC patients when compared with their expression levels in the AEH group (data not shown). The healthy donor group 

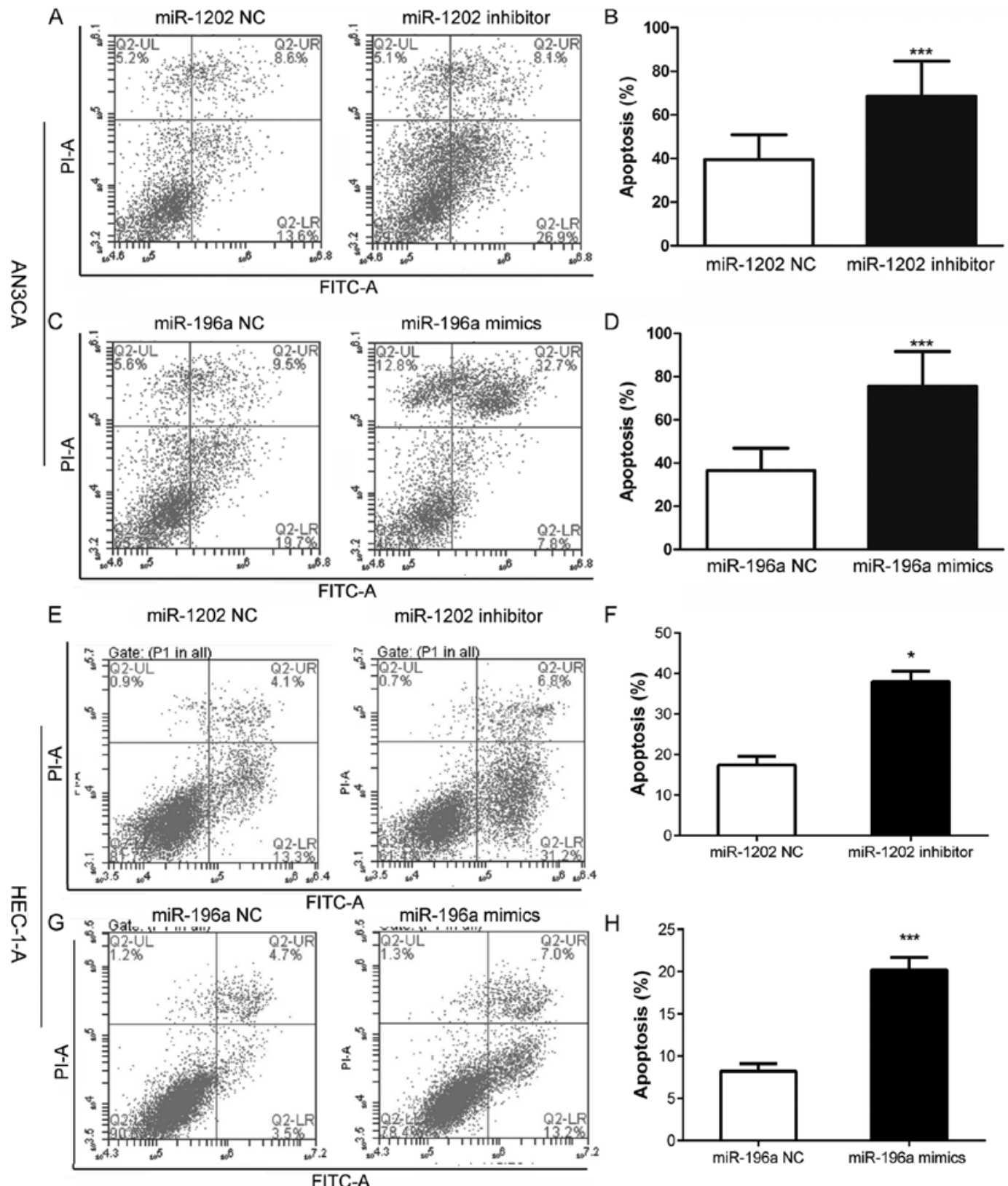

Figure 3. Effects of miR-1202 and miR-196a on cell apoptosis after pre-treatment. (A and B) AN3CA cells were transfected with miR-1202 inhibitor for $72 \mathrm{~h}$, and then assayed to determine the percentage of apoptotic cells. (C and D) AN3CA cells were transfected with miR-196a mimics for $72 \mathrm{~h}$ prior to being assayed for apoptosis. (E and F) HEC-1-A cells were transfected with miR-1202 inhibitor for $72 \mathrm{~h}$, and then assayed to determine the percentage of apoptotic cells. (G and H) HEC-1-A cells were transfected with miR-196a mimics for $72 \mathrm{~h}$ prior to being assayed for apoptosis. Data are expressed as the mean \pm standard deviation. All data represent results from 3 independent experiments; $\mathrm{P}<0.05,{ }^{* * * *} \mathrm{P}<0.001$ vs. a control group assayed at same time-point. AN3CA and HEC-1-A cells transfected with miR-1202 inhibitor or miR-196a mimics served as the control groups.

served as a negative control group. A correlation analysis of hsa-miRNA expression in the tissue samples indicated that all of the hsa-miRNAs were expressed at significantly different levels in the 3 groups (Fig. 1).

Verification and selection of differentially expressed miRNAs in tissue specimens. To confirm the results obtained from the miRNA microarray assay, the expression levels of 12 miRNAs which had been analyzed by microarray were further analyzed by real-time PCR. Our results revealed that when compared with their expression levels in the healthy group, hsa-miR-5787, hsa-miR-6749-5p and hsa-miR-1202 were expressed at successively increased levels in tissue and blood samples (Fig. 2B, D and F), while levels of hsa-miR-338-3p, hsa-miR-449a, hsa-miR-196a were successively downregulated in tissue and blood samples, respectively, from patients of the AEH and EC group (Fig. 2A, C and E). Thus, the real-time PCR results were consistent with the microarray assay results. Among these various candidates, we selected miR-1202 and miR-196a as our target mRNAs for further experiments.

Effects of miR-1202 and miR-196a on cell apoptosis. An Annexin V-FITC/PI staining analysis indicated that AN3CA cells transfected with miR-1202 inhibitor (Fig. 3A and B) or miR-196a mimics (Fig. 3C and D), respectively, for $72 \mathrm{~h}$ had 

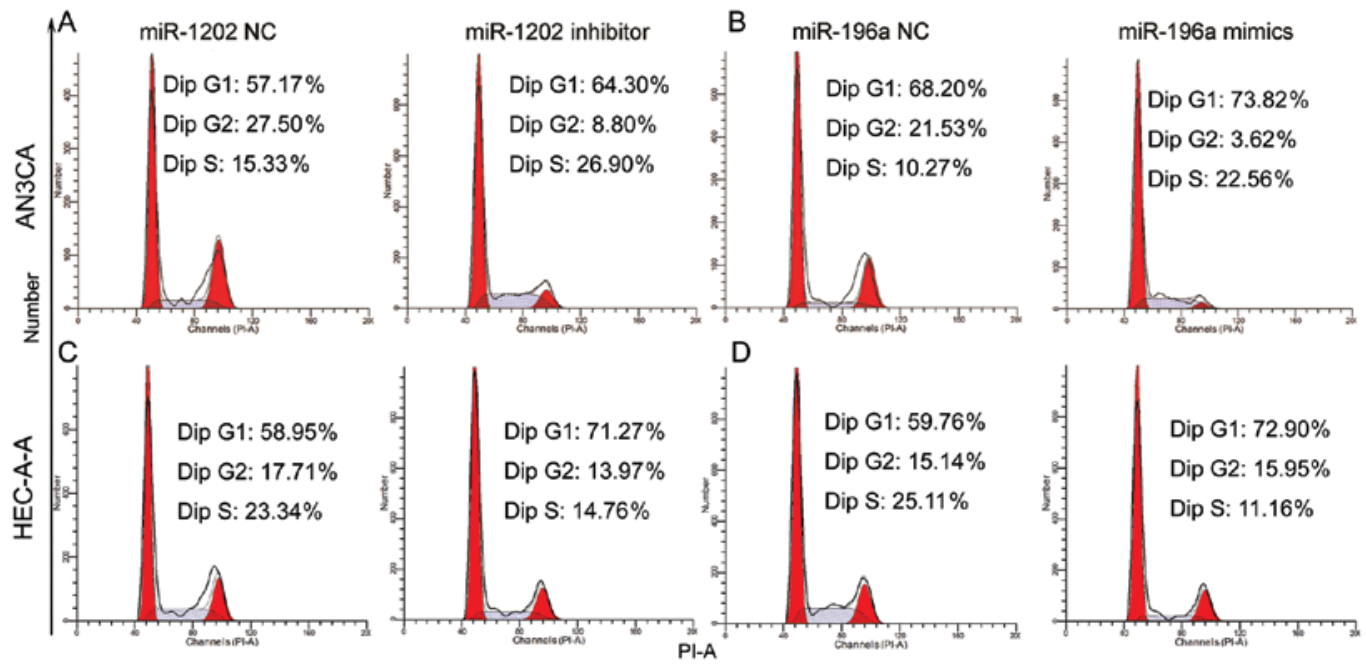

Figure 4. Effects of miR-1202 and miR-196a on the cell cycle distribution after pre-treatment. (A and C) AN3CA and HEC-1-A cells were transfected with a miR-1202 inhibitor for $72 \mathrm{~h}$ and then examined for the percentage of cells in different phases of the cell cycle. (B and D) AN3CA and HEC-1-A cells were transfected with miR-196a mimics for $72 \mathrm{~h}$ and then examined for the percentage of cells in different phases of the cell cycle. Data are expressed as the mean \pm standard deviation. All data represent results from 3 independent experiments.

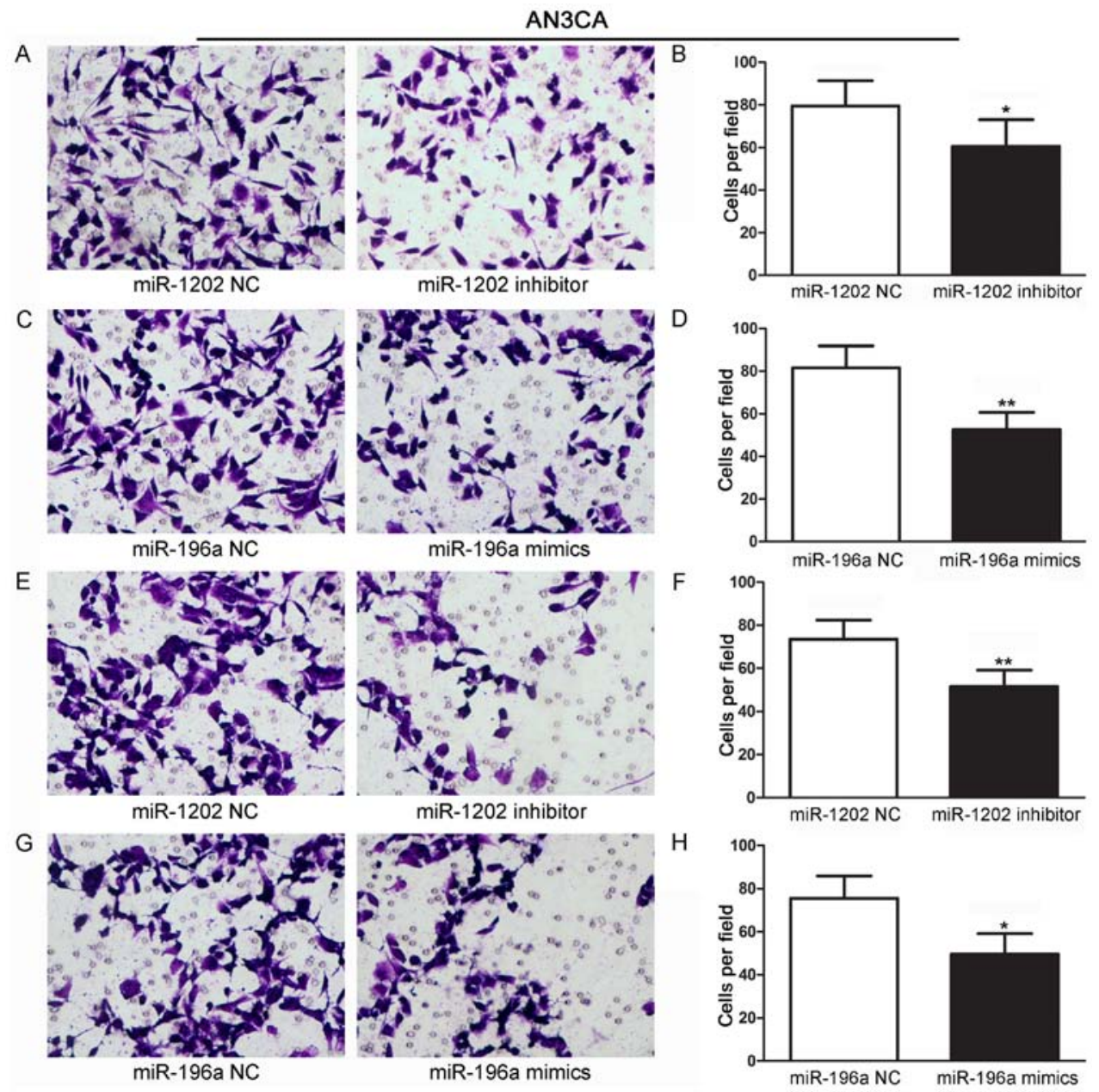

Figure 5. Effects of miR-1202 and miR-196a on cell migration and cell invasion after pre-treatment in the AN3CA cell line. (A) AN3CA cells were transfected with a miR-1202 inhibitor, and then assessed in migration assays that used Transwell inserts without basement membrane extract. (B) The percentage of AN3CA cells that exhibited migration at $24 \mathrm{~h}$ after transfection with the miR-1202 inhibitor. (C) AN3CA cells were transfected with miR-196a mimics, and then assessed in migration assays that used Transwell inserts without basement membrane extract. (D) The percentage of AN3CA cells that exhibited migration at $24 \mathrm{~h}$ after transfection with miR-196a mimics. (E) AN3CA cells were transfected with a miR-1202 inhibitor, and then assessed in invasion assays that used Transwell inserts without basement membrane extract. (F) The percentage of invasive AN3CA cells at $24 \mathrm{~h}$ after transfection with a miR-1202 inhibitor. (G) Cell invasion assays were performed using Transwell inserts without basement membrane extract after the cells had been transfected with miR-196a mimics. (H) The percentage of invasive AN3CA cells at $24 \mathrm{~h}$ after transfection with miR-196a mimics. Magnification, x200. Cells were stained with Giemsa; ${ }^{*} \mathrm{P}<0.05$ vs. a control group examined at the same time-point; ${ }^{* *} \mathrm{P}<0.01$ vs. a normal control group examined at the same time-point. 
HEC-1A

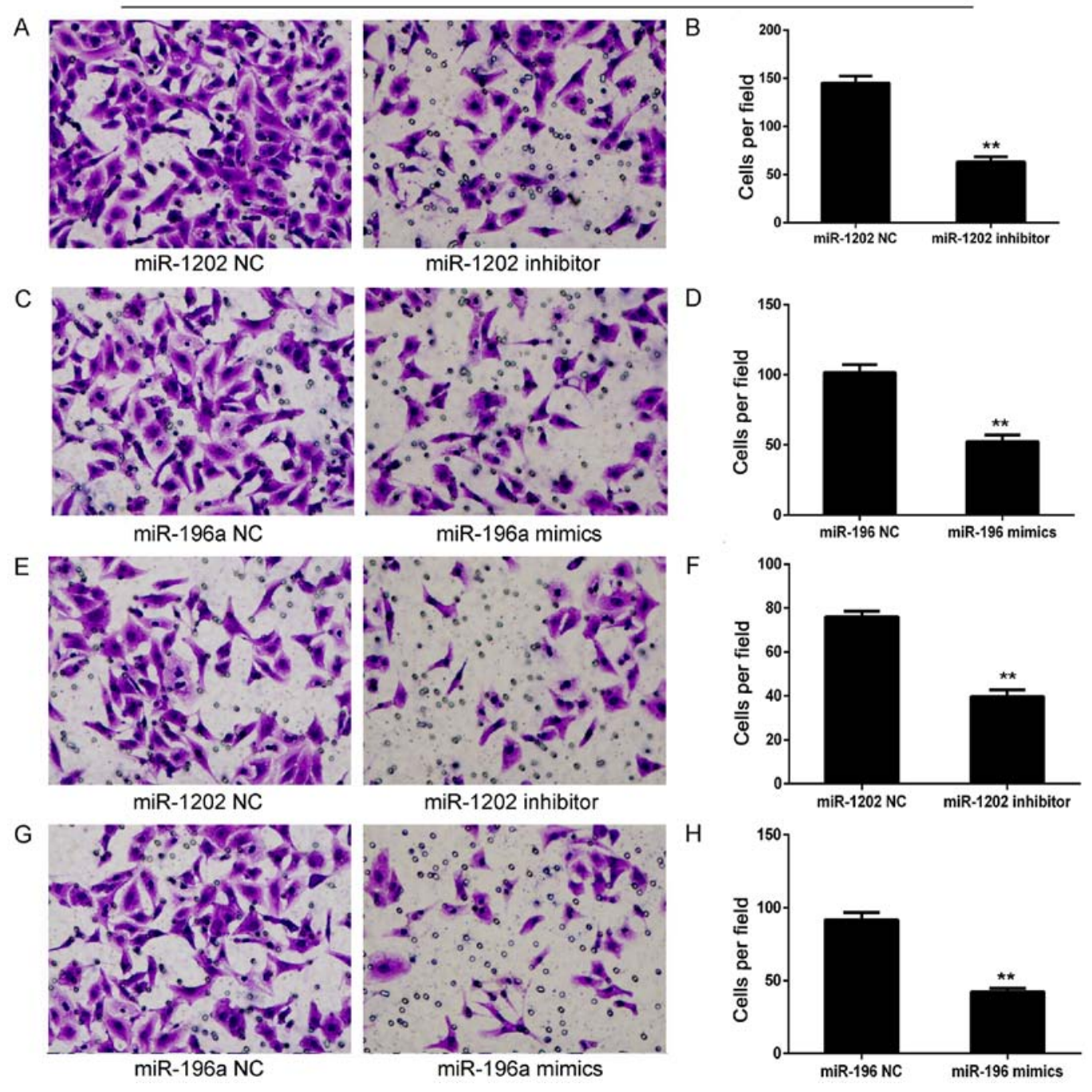

Figure 6. Effects of miR-1202 and miR-196a on cell migration and cell invasion after pre-treatment in HEC-1-A cell line. (A) HEC-1-A cells were transfected with a miR-1202 inhibitor. (B) The percentage of HEC-1-A cells that exhibited migration at $24 \mathrm{~h}$ after transfection with the miR-1202 inhibitor. (C) HEC-1-A cells were transfected with miR-196a mimics. (D) The percentage of HEC-1-A cells that exhibited migration at $24 \mathrm{~h}$ after transfection with miR-196a mimics. (E) HEC-1-A cells were transfected with a miR-1202 inhibitor. (F) The percentage of invasive HEC-1-A cells at $24 \mathrm{~h}$ after transfection with a miR-1202 inhibitor. (G) Cell invasion assays were performed using Transwell inserts without basement membrane extract after the cells had been transfected with miR-196a mimics. (H) The percentage of invasive HEC-1-A cells at $24 \mathrm{~h}$ after transfection with miR-196a mimics. Magnification, x200. Cells were stained with Giemsa; ${ }^{\mathrm{P}}<0.05$ vs. a control group examined at the same time-point; ${ }^{* *} \mathrm{P}<0.01$ vs. a normal control group examined at the same time-point.

significantly increased levels of apoptosis and necrosis, as indicated by the presence of a prominentsub-G1 peak (apoptotic cells). The rates of apoptosis and necrosis were significantly increased to $60 \%$ after $72 \mathrm{~h}$ of treatment, and were higher than in either of the two NC groups. Similarly, we performed the same assessment in the HEC-1-A cells (Fig. 3E-H). Treatment with miR-1202 inhibitor (Fig. 3E and F) and miR-196a mimics (Fig. 3G and H), respectively, for $72 \mathrm{~h}$ resulted in a significant increase in apoptosis and necrosis in the HEC-1-A cells shown as a significant sub-G1 peak (apoptotic cells).

Effects of miR-1202 and miR-196a on cell cycle distribution. We performed flow cytometric assays to assess the effect of miR-1202 silencing or miR-196a overexpression on the AN3CA and HEC-1-A cell cycle. After transfection with miR-1202 inhibitor (Fig. 4A and C) or miR-196a mimics (Fig. 4B and D) for $72 \mathrm{~h}$, the percentage of G0/G1 phase cells was significantly increased, while the percentage of cells in the $\mathrm{G} 2 / \mathrm{M}$ phases decreased.

Effects of miR-1202 and miR-196a on cell migration and invasion abilities. We investigated the roles of miR-1202 and miR-196a in mediating EC cell migration and invasion. AN3CA cells that had been transfected with miR-1202 inhibitor or miR-196a mimics were evaluated for their migration and invasion abilities. AN3CA cells transfected with scrambled miRNA inhibitors or mimics were used as NC groups. The results revealed that $\mathrm{AN} 3 \mathrm{CA}$ and $\mathrm{HEC}-1-\mathrm{A}$ cells transfected with miR-1202 inhibitor (Figs. 5A and B, and 6A and B) or miR-196a mimics (Figs. 5C and D, and 6C and D) displayed a significantly decreased migratory ability when compared with the NC groups $(\mathrm{P}<0.01)$. In parallel, AN3CA and HEC-1-A cells transfected with miR-1202 inhibitor (Figs. 5E and F, and 6E and F) or miR-196a mimics (Figs. 5G and $\mathrm{H}$, and $6 \mathrm{G}$ and $\mathrm{H}$ ) also displayed a significantly decreased invasion ability $(\mathrm{P}<0.01)$. 


\section{Discussion}

Microarray panels have been described as a new and powerful methodology for identifying miRNA expression patterns that may distinguish between samples of carcinoma and non-carcinoma tissue $(18,19)$. However, no study has described the miRNA microarray profile of endometrial cancer (EC) tissue, and particularly tissue in which EC is accompanied by AEH. In the present study, we identified some global changes in miRNA expression that characterize the difference between human malignant and non-malignant tissue samples. The consistency of our findings was supported by our use of TaqMan qRT-PCR methodology to validate 12 of the most significant differentially expressed miRNAs in an extended series of human tissue samples. Finally, highly expressed miR-1202 and lowly expressed miR-196a were selected as our research candidates for further investigation. Next, we explored the effects of these two miRNAs on cellular functions in vitro, and found that miR-1202 may protect AN3CA cells against apoptosis and increase their $\mathrm{S}$ phase arrest, while miR-196a may reverse these effects. Collectively, our findings indicate the importance of miR-1202 and miR-196a in the pathogenesis of $\mathrm{EC}$, and contribute to the understanding of the miRNA-driven pathways related to EC.

In agreement with our results, a previous study revealed that miR-1202 was strongly correlated with a shorter overall survival time among patients with adrenocortical carcinoma (ACC) (20). Moreover, other studies have shown that high levels of miR-1202 in human brain tissue may play an important role in the pathophysiology of depression, suggesting miR-1202 as a potential target for novel anti-depressant agents $(21,22)$. Additionally, overexpression of miR-1202 may be associated with lymph node metastasis (23). In the present study, we demonstrated that miR-1202 expression was significantly higher in EC patients than in AEH patients and a healthy donor group, suggesting that miR-1202 inhibitor may exert a protective effect in EC. We also demonstrated that miR-196a expression levels were significantly lower in specimens of EC tissue than in specimens of endometrial tissue obtained from AEH patients or normal control subjects. This supports previous studies that suggested miR-196a as a newly discovered promising biomarker for tumor progression (24-27). However, its dysregulation in endometrioid endometrial carcinoma (EEC), revealed that miR-196a may also work synergistically with other miRNAs thought to be involved in various diseases (28). In cervical cancer (26) and head and neck squamous cell carcinoma (29), miR-196a was overexpressed in the absence of HOXC8/HOXB9 expression, which suggests its role as an oncomiR. Regarding our research candidates, miR-1202 and miR-196a, further investigation may be warranted to explore the regulation mechanism even if numerous studies have revealed that both of them may negatively regulate target genes through binding to the $3^{\prime}$ UTR of their target mRNAs, such as SOX11/12 (30), MAP3K1 (31), PCDH17 (32) and TP53 (33).

The present study has several limitations that should be mentioned. First, the study was performed using native human tissues, and false-positive results may exist due to the limited sample size. Thus, only miRNAs that revealed a significant aberrant change were included in the present study. Second, the molecular mechanism by which alterations in miRNAs cause $\mathrm{EC}$ or $\mathrm{AEH}$ has not identified, and requires further bioinformatic analysis and validation testing. A better understanding of the bio-functional significance of broad and often subtle variations in miRNA levels which occur during development of EC would allow specific-mRNA molecules to be identified for manipulation in cases of EC, and thus, lead to new therapeutic interventions that are based on rational target selection.

In conclusion, the present study identified the miRNA profile signature in 3 different cohorts: EC and AEH patients, and healthy donors. This pattern of aberrantly expressed miRNAs may contribute to our understanding of EC and/or AEH. Our findings suggest that silencing of miR-1202 or overexpression of miR-196a may increase cell apoptosis and G1 phase arrest in EC cells with lower migration and invasion abilities. Additionally, the miRNA expression signature described in the present study can aid in developing new molecular-based therapies for EC.

\section{Acknowledgements}

The present study was funded by the Natural Science Foundation of GuangXi (no. 2013GXNSFAA01956).

\section{References}

1. Braun MM, Overbeek-Wager EA and Grumbo RJ: Diagnosis and management of endometrial cancer. Am Fam Physician 93: 468-474, 2016.

2. Prat J, Gallardo A, Cuatrecasas M and Catasús L: Endometrial carcinoma: Pathology and genetics. Pathology 39: 72-87, 2007.

3. Uharcek P: Prognostic factors in endometrial carcinoma. J Obstet Gynaecol Res 34: 776-783, 2008.

4. Rossouw JE, Anderson GL, Prentice RL, LaCroix AZ, Kooperberg C, Stefanick ML, Jackson RD, Beresford SA, Howard BV, Johnson KC, et al; Writing Group for the Women's Health Initiative Investigators: Risks and benefits of estrogen plus progestin in healthy postmenopausal women: Principal results From the Women's Health Initiative randomized controlled trial. JAMA 288: 321-333, 2002.

5. Corrado G, Baiocco E, Carosi M and Vizza E: Progression of conservatively treated endometrial complex atypical hyperplasia in a young woman: A case report. Fertil Steril 90: 2006.e5-2006.e8, 2008.

6. Ofinran $\mathrm{O}$ and Balega $\mathrm{J}$ : The value of magnetic resonance imaging in investigating complex atypical hyperplasia of the endometrium. Minerva Ginecol 68: 400-404, 2016.

7. Jurcevic S, Olsson B and Klinga-Levan K: MicroRNA expression in human endometrial adenocarcinoma. Cancer Cell Int 14: 88, 2014.

8. Yang S, Jia Y, Liu X, Winters C, Wang X, Zhang Y, Devor EJ, Hovey AM, Reyes HD, Xiao X, et al: Systematic dissection of the mechanisms underlying progesterone receptor downregulation in endometrial cancer. Oncotarget 5: 9783-9797, 2014.

9. Torres A, Torres K, Wdowiak P, Paszkowski T and Maciejewski R: Selection and validation of endogenous controls for microRNA expression studies in endometrioid endometrial cancer tissues. Gynecol Oncol 130: 588-594, 2013.

10. Santulli G: MicroRNAs and endothelial (Dys) function. J Cell Physiol 231: 1638-1644, 2016.

11. Jiang C, Chen X, Alattar M, Wei J and Liu H: MicroRNAs in tumorigenesis, metastasis, diagnosis and prognosis of gastric cancer. Cancer Gene Ther 22: 291-301, 2015.

12. Widodo, Djati MS and Rifa'i M: Role of MicroRNAs in carcinogenesis that potential for biomarker of endometrial cancer. Ann Med Surg 7: 9-13, 2016.

13. Boren T, Xiong Y, Hakam A, Wenham R, Apte S, Wei Z, Kamath S, Chen DT, Dressman H and Lancaster JM: MicroRNAs and their target messenger RNAs associated with endometrial carcinogenesis. Gynecol Oncol 110: 206-215, 2008. 
14. Xu YY,Wu HJ,MaHD, Xu LP,Huo Y and Yin LR: MicroRNA-503 suppresses proliferation and cell-cycle progression of endometrioid endometrial cancer by negatively regulating cyclin D1. FEBS J 280: 3768-3779, 2013.

15. Kalia M: Biomarkers for personalized oncology: Recent advances and future challenges. Metabolism 64 (Suppl 1): S16-S21, 2015.

16. Lee TS, Jeon HW, Kim YB, Kim YA, Kim MA and Kang SB: Aberrant microRNA expression in endometrial carcinoma using formalin-fixed paraffin-embedded (FFPE) tissues. PLoS One 8: e81421, 2013.

17. Luna-Aguirre CM, de la Luz Martinez-Fierro M, Mar-Aguilar F, Garza-Veloz I, Treviño-Alvarado V, Rojas-Martinez A, Jaime-Perez JC, Malagon-Santiago GI, Gutierrez-Aguirre CH, Gonzalez-Llano $\mathrm{O}$, et al: Circulating microRNA expression profile in B-cell acute lymphoblastic leukemia. Cancer Biomark 15: 299-310, 2015.

18. Pellatt DF, Stevens JR, Wolff RK, Mullany LE, Herrick JS, Samowitz W and Slattery ml: Expression profiles of miRNA subsets distinguish human colorectal carcinoma and normal colonic mucosa. Clin Transl Gastroenterol 7: e152, 2016.

19. Plieskatt JL, Rinaldi G, Feng Y, Levine PH, Easley S, Martinez E, Hashmi S, Sadeghi N, Brindley PJ, Bethony JM, et al: Methods and matrices: Approaches to identifying miRNAs for nasopharyngeal carcinoma. J Transl Med 12: 3, 2014.

20. Özata DM, Caramuta S, Velázquez-Fernández D, Akçakaya $P$ Xie H, Höög A, Zedenius J, Bäckdahl M, Larsson C and Lui WO The role of microRNA deregulation in the pathogenesis of adrenocortical carcinoma. Endocr Relat Cancer 18: 643-655, 2011.

21. Rucker JJ and McGuffin P: Chipping away at major depressive disorder. Genome Biol 15: 421, 2014.

22. Lopez JP, Lim R, Cruceanu C, Crapper L, Fasano C, Labonte B, Maussion G, Yang JP, Yerko V, Vigneault E, et al: miR-1202 is a primate-specific and brain-enriched microRNA involved in major depression and antidepressant treatment. Nat Med 20: 764-768, 2014

23. Wang Z, Zhang H, Zhang P, Li J, Shan Z and Teng W: Upregulation of miR-2861 and miR-451 expression in papillary thyroid carcinoma with lymph node metastasis. Med Oncol 30: 577,2013
24. Chen ZF, Ma LL and Xue HB: Common polymorphisms of the microRNA genes (miR-146a and miR-196a-2) and gastric cancer risk: An updated meta-analysis. Genet Mol Res 14: 8589-8601, 2015.

25. Li Y, Jin L, Chen D, Liu J, Su Z, Yang S, Gui Y, Mao X, Nie G and Lai Y: Tumor suppressive miR-196a is associated with cellular migration, proliferation and apoptosis in renal cell carcinoma. Mol Med Rep 14: 560-566, 2016.

26. Liu P, Xin F and Ma CF: Clinical significance of serum miR-196a in cervical intraepithelial neoplasia and cervical cancer. Genet Mol Res 14: 17995-18002, 2015.

27. Fan Y, Fan J, Huang L, Ye M, Huang Z, Wang Y, Li Q and Huang J: Increased expression of microRNA-196a predicts poor prognosis in human ovarian carcinoma. Int J Clin Exp Pathol 8: 4132-4137, 2015

28. Xiong H, Li Q, Liu S, Wang F, Xiong Z, Chen J, Chen H, Yang Y, Tan X, Luo Q, et al: Integrated microRNA and mRNA transcriptome sequencing reveals the potential roles of miRNAs in stage I endometrioid endometrial carcinoma. PLoS One 9: e110163, 2014.

29. Darda L, Hakami F, Morgan R, Murdoch C, Lambert DW and Hunter KD: The role of HOXB9 and miR-196a in head and neck squamous cell carcinoma. PLoS One 10: e0122285, 2015.

30. Roisman A, Huamán Garaicoa F, Metrebian F, Narbaitz M, Kohan D, García Rivello H, Fernandez I, Pavlovsky A, Pavlovsky M, Hernández L, et al: SOXC and MiR17-92 gene expression profiling defines two subgroups with different clinical outcome in mantle cell lymphoma. Genes Chromosomes Cancer 55: 531-540, 2016

31. Liu C, Wang S, Zhu S, Wang H, Gu J, Gui Z, Jing J, Hou X and Shao Y: MAP3K1-targeting therapeutic artificial miRNA suppresses the growth and invasion of breast cancer in vivo and in vitro. Springerplus 5: 11, 2016.

32. Zhang X, Yao X, Qin C, Luo P and Zhang J: Investigation of the molecular mechanisms underlying metastasis in prostate cancer by gene expression profiling. Exp Ther Med 12: 925-932, 2016.

33. Zhu M, Xu Z, Wang K, Wang N and Li Y: microRNA and gene networks in human pancreatic cancer. Oncol Lett 6: 1133-1139, 2013. 